SCHOOL OF OPERATIONS RESEARCH AND INDUSTRIAL ENGINEERING COLLEGE OF ENGINEERING CORNELL UNIVERSITY

ITHACA, NY 14853-3801

TECHNICAL REPORT NO. 1143

June 1995

\title{
Classes of \\ Mixing Stable Processes
}

by

J. Rosiński and G. Samorodnitsky 


\title{
Classes of Mixing Stable Processes*
}

\author{
Jan Rosiński \\ Department of Mathematics \\ University of Tennessee \\ Knoxville, TN 37996
}

\author{
Gennady Samorodnitsky \\ School of OR/IE \\ Cornell University \\ Ithaca, NY 14853
}

June 26, 1995

\begin{abstract}
Every measurable stationary $\alpha$-stable process with $0<\alpha<2$ can be related to a nonsingular flow on a $\sigma$-finite measure space. We establish the relationship between properties of the flow and mixing of the stationary stable process. We provide the first example of a mixing stationary stable process corresponding to a conservative flow. We show further the connection between the expected return time of the flow to sets of finite positive measure and the mixing properties of the process.
\end{abstract}

\footnotetext{
${ }^{*}$ Rosinski's research was supported in part by the NSF grant DMS-94-06294 at the University of Tennessee at Knoxville. Samorodnitsky's research was supported by the NSF grant DMS-9400535, the NSA grant MDA904-95-H-1036 at Cornell University and United States - Israel Binational Science Foundation (BSF) Grant 92-00074/1. Part of Samorodnitsky's research was done at Chalmers University, Göteborg, under support of Swedish Natural Science Research Council grant F-GF 09207-305, while the other part was done at ETH Zürich, under support of Forschungsinstitut für Mathematik and Institut für Operations Research.

Key Words and phrases: stable processes, stationary processes, ergodicity, mixing, nonsingular flow, dissipative and conservative flows, spectral representation, expected return time, asymptotic singularity, positive and null recurrence AMS (1991) Subject Classification: 60E07, 60G10, 28D10
} 


\section{Introduction}

Let $\boldsymbol{X}=\left\{X_{t}: t \in \mathbf{T}\right\}$ be a stationary symmetric $\alpha$-stable (S $\alpha \mathrm{S}$ ) process, where $0<\alpha<2$ and $\mathbf{T}=\mathbf{Z}$ or $\mathbf{R}$. It is well known that $\boldsymbol{X}$ has a stochastic integral representation

$$
\left\{X_{t}, t \in \mathbf{T}\right\} \stackrel{\mathrm{d}}{=}\left\{\int_{S} f_{t} d M, t \in \mathbf{T}\right\}
$$

where $M$ is an independently scattered $\mathrm{S} \alpha \mathrm{S}$ random measure on some Lebesgue space $(S, \mathcal{B}, \mu)$ and $\left\{f_{t}\right\}_{t \in \mathbf{T}}$ is the orbit of a one-parameter group $\{U\}_{t \in \mathbf{T}}$ of linear isometries on a subspace of $L^{\alpha}(S, \mathcal{B}, \mu)$, i.e. $f_{t}=U^{t} f$ for some $f \in L^{\alpha}(S, \mathcal{B}, \mu)$ (see [Har82] or [ST94]). "르" means "equal in distribution". Furthermore, it was shown in [Ros95] that one can choose $f_{t}$ 's of the form

$$
f_{t}=a_{t}\left(\frac{d\left(\mu \circ \phi_{t}\right)}{d \mu}\right)^{1 / \alpha} f \circ \phi_{t}, t \in \mathbf{T}
$$

where $a_{t}: S \rightarrow\{|z|=1\}\left(a_{t} \in\{-1,1\}\right.$ if $\boldsymbol{X}$ is real) satisfies the equation $a_{t_{1}+t_{2}}=$ $a_{t_{2}} \cdot a_{t_{1}} \circ \phi_{t_{2}} \mu$-a.e. for every $t_{1}, t_{2} \in \mathbf{T}, \phi_{t}: S \rightarrow S$ is a nonsingular flow on $(S, \mathcal{B}, \mu)$, and $f \in L^{\alpha}(S, \mathcal{B}, \mu)$. It is easy to see that in the case $\mathbf{T}=\mathbf{Z}$ one has $\phi_{t}=V^{t}$, for some nonsingular map $V: S \rightarrow S$, and $a_{t}$ are determined by $a_{1}$ and $V$.

Since the theory of nonsingular flows on measure spaces is so well developed, an obvious direction of research for probabilists with interest in stable processes is to study the way the properties of the flow determine the properties of the corresponding stable process as well as to identify flows which generate specific classes of stable processes. Our goal here is to relate the ergodic properties of the stationary stable process to the properties of the underlying flow.

The study of ergodic properties of stationary stable (in fact, of general infinitely divisible processes) processes begins with Maruyama [Mar70], who showed that mixing property for the latter is equivalent to weak convergence of the two-dimensional distributions of the process to a limit with independent marginals, when the time lag increases to infinity. Apparently unaware of this work, Cambanis, Hardin and Weron [CHW87] suggested another, somewhat more complicated, set of necessary and sufficient conditions for mixing (and ergodicity) of stationary stable processes. Treating ergodicity has become easier since the result of Podgorski [Pod92] showing that for the latter ergodicity and weak mixing are equivalent. Finally, Gross [Gro94] has simplified necessary and sufficient conditions for mixing of stationary stable processes even further by showing that for those one does not even need to check the whole of Maruyama's assumptions. It is the work of Gross that served as the starting point for the present research.

Obviously one can assume that the the smallest set supporting all $f_{t}$ 's in (1.1) is $S$, i.e.,

$$
\operatorname{supp}\left\{f_{t}: t \in \mathbf{T}\right\}=S \quad \mu-a . e .
$$


Otherwise, we can just reduce the space of integration accordingly. The assumption (1.3) will simplify our formulations considerably, and so this assumption will be in effect throughout this paper, unless mentioned otherwise.

Any stationary $\mathrm{S} \alpha \mathrm{S}$ process can be decomposed in the form

$$
\boldsymbol{X} \stackrel{\mathrm{d}}{=} \boldsymbol{X}^{1}+\boldsymbol{X}^{2}+\boldsymbol{X}^{3}
$$

(see Rosinski [Ros95]), where $\boldsymbol{X}^{1}, \boldsymbol{X}^{2}$, and $\boldsymbol{X}^{3}$ are mutually independent stationary $\mathrm{S} \alpha \mathrm{S}$ processes such that $\boldsymbol{X}^{1}$ is a superposition of moving averages (or a mixed moving average process), $\boldsymbol{X}^{2}$ is harmonizable, and $\boldsymbol{X}^{3}$ does not admit moving average or harmonizable components. The process $\boldsymbol{X}^{1}$ is generated by a dissipative flow, while the other two processes in the above decomposition are generated by infinitely recurrent flows. Furthermore, the harmonizable process $\boldsymbol{X}^{2}$ is essentially generated by the identity flow. Process $\boldsymbol{X}^{3}$ itself has a stochastic integral representation of the form (1.1)-(1.2) and the flow $\left\{\phi_{t}\right\}$ in this case is infinitely recurrent and does not have fixed points ([Ros95]). It turns out that $\boldsymbol{X}^{1}$ is always mixing [SRMC93] and $\boldsymbol{X}^{2}$ is never mixing (provided $\boldsymbol{X}^{2} \neq 0$ ) [Mar70]. In fact, this corresponds perfectly well to our intuition, because the fact that mixed moving averages are generated by dissipative flows implies that the observations of the process $\boldsymbol{X}^{\mathbf{1}}$ at remote instances of time are generated by integration over nearly disjoint sets - and the random measure $M$ in (1.1) is independently scattered. Therefore, one feels that these observations are nearly independent, and so expect mixing. On the other hand, infinitely recurrent flows produce processes for which integration over the same sets plays an important role for time points far apart, and so the process "remembers" much from its past. The extreme case is, of course, that of the identity flows, and that's why it is not surprising that harmonizable processes are not even ergodic (the latter is true in every infinitely divisible non-Gaussian case by a result of Maruyama [Mar70]).

By the same token one would not expect that many of the processes of the type $\boldsymbol{X}^{3}$ were mixing. Indeed, A. Gross [Gro94] has shown that a large class of processes of this type consisting of the so-called doubly stationary process with finite control measure $\mu$ are not mixing (nor ergodic). This is clear also because such processes share with the harmonizable ones the property of being a non-trivial mixture of stationary processes (via their series representation), and so they cannot be ergodic. However, this implies that the class of mixing $S \alpha S$ processes of the type $\boldsymbol{X}^{3}$ is rather small, and until now it was not even known whether or not this class was not empty, for the only known examples of mixing (or ergodic) $\mathrm{S} \alpha \mathrm{S}$ processes known so far were superpositions of moving averages, i.e., processes of type $\boldsymbol{X}^{1}$.

In this note we introduce a new class of mixing $S \alpha S$ processes, consisting of processes of type $\boldsymbol{X}^{3}$. This class is obtained by considering Markov flows $\left\{\phi_{t}\right\}$ in (1.2) with infinite stationary initial distributions $m$ (see Section 3). A typical example of such a flow is the shift transformation for a symmetric recurrent random walk on $\mathbf{R}$ starting from a point chosen accordingly to the Lebesgue measure. This establishes an 
interesting connection between Markov and stable processes. In the case of recurrent Markov chains infinite invariant measures correspond to null recurrence, or Markov chains for which expected return time to the initial state is infinite. We will see that in general, a conservative flow corresponding to an ergodic stable process must take an expected infinite time to return to sets of finite positive measure.

In Section 2 we will show that mixing and ergodicity of $\mathrm{S} \alpha \mathrm{S}$ processes are entirely determined by properties of the flow $\left\{\phi_{t}\right\}$ in (1.2). We identify these properties and provide some conditions characterizing mixing and ergodicity in terms of $\phi_{t}$ and $\mu$, which complement conditions in [Gro94].

\section{Conditions for mixing and ergodicity}

Recall that a stationary process $\boldsymbol{X}$ is said to be ergodic if

$$
\lim _{\tau \rightarrow \infty} \frac{1}{\tau} \int_{0}^{\tau} P\left(A \cap S^{t} B\right) d t=P(A) P(B)
$$

for any $A, B \in \mathcal{F}_{\boldsymbol{X}}$, where $\mathcal{F}_{\boldsymbol{X}}$ is the $\sigma$-field generated by $\boldsymbol{X}$ and $S^{t}$ is the corresponding shift transformation (in the discrete-time, the integral is replaced by a sum). $\boldsymbol{X}$ is said to be mixing if

$$
\lim _{t \rightarrow \infty} P\left(A \cap S^{t} B\right)=P(A) P(B),
$$

for any $A, B \in \mathcal{F}_{\boldsymbol{X}} \cdot \boldsymbol{X}$ is said to be weakly mixing if the above limit holds with $t$ is restricted to a set of density one which may depend on $A$ and $B$. Alternatively, $\boldsymbol{X}$ is weakly mixing if

$$
\lim _{\tau \rightarrow \infty} \frac{1}{\tau} \int_{0}^{\tau}\left|P\left(A \cap S^{t} B\right)-P(A) P(B)\right| d t=0
$$

for any $A, B \in \mathcal{F} \boldsymbol{X}$, which shows that weak mixing is an intermediate property between ergodicity and mixing (see [Pet83]). However, for $\mathrm{S} \alpha \mathrm{S}$ processes ergodicity and weak mixing are equivalent by [Pod92]. It is also known that there are non-mixing ergodic (and thus weakly mixing) stationary $\mathrm{S} \alpha \mathrm{S}$ processes (see [GR93]).

In this section we will establish a one-to-one correspondence between ergodic properties of a stochastic process $\boldsymbol{X}$ and certain asymptotic properties of the flow $\left\{\phi_{t}\right\}$ in (1.2). We will need the following definitions. A measurable flow $\left\{\phi_{t}\right\}_{t \in \mathbf{T}}$ on a finite measure space $(S, \mathcal{B}, \mu)$ is said to be asymptotically singular if $\forall \epsilon>0 \exists t_{0}$ $\forall|t|>t_{0} \exists A=A(t, \epsilon) \in \mathcal{B}$ such that

$$
\mu(A)<\epsilon \text { and } \mu\left(\phi_{t} A\right)>\mu(S)-\epsilon .
$$

It is trivial that an asymptotically singular flow is not $\mu$-preserving, moreover, there can not be any finite $\phi_{t}$-preserving measure $\nu$ equivalent to $\mu$. The usual tool in a 
study of singularity/regularity of measures is the Hellinger integral. It is defined for finite measures $\mu$ and $\nu$ by

$$
H(\mu, \nu)=\int_{S} \sqrt{\frac{d \mu}{d \lambda} \frac{d \nu}{d \lambda}} d \lambda,
$$

where $\lambda$ is certain (or any) finite measure such that $\mu \ll \lambda$ and $\nu \ll \lambda$. A convenient (but somewhat loose) way of writing (2.2) is

$$
H(\mu, \nu)=\int_{S} \sqrt{d \mu d \nu}
$$

It is easy to show that $\left\{\phi_{t}\right\}_{t \in \mathbf{T}}$ is asymptotically singular with respect to $\mu$ if and only if

$$
\lim _{|t| \rightarrow \infty} H\left(\mu, \mu \circ \phi_{t}\right)=0
$$

We will need the following characterization.

Proposition 2.1 Let $\boldsymbol{X}=\left\{X_{t}\right\}_{t \in \mathbf{T}}$ be a stationary $S \alpha S$ process with representation $\left\{f_{t}\right\}_{t \in \mathbf{T}}$ satisfying (1.1). $\boldsymbol{X}$ is mixing if and only if for some (equivalently, any) $\delta \in(0,1)$

$$
\lim _{t \rightarrow \infty} \int_{S}\left|f_{0}\right|^{\alpha \delta}\left|f_{t}\right|^{\alpha(1-\delta)} d \mu=0
$$

Proof: By a result of A. Gross ([Gro94], Theorem 2.7) $\boldsymbol{X}$ is mixing if and only if

$$
\mu\left\{f_{0} \in K,\left|f_{t}\right|>\epsilon\right\} \rightarrow 0
$$

for every compact set $K \subset \mathbf{R}$ bounded away from 0 and $\epsilon>0$. Suppose that $\boldsymbol{X}$ is mixing. We have for any $\eta \in(0,1)$ and $\epsilon>0$ (that will be chosen later)

$$
\begin{aligned}
& \int_{S}\left|f_{0}\right|^{\alpha \delta}\left|f_{t}\right|^{\alpha(1-\delta)} d \mu=\int_{\left|f_{0}\right| \in\left[\eta, \eta^{-1}\right],\left|f_{t}\right|>\epsilon}\left|f_{0}\right|^{\alpha \delta}\left|f_{t}\right|^{\alpha(1-\delta)} d \mu \\
& +\int_{\left|f_{0}\right| \notin\left[\eta, \eta^{-1}\right]}\left|f_{0}\right|^{\alpha \delta}\left|f_{t}\right|^{\alpha(1-\delta)} d \mu+\int_{\left|f_{0}\right| \in\left[\eta, \eta^{-1}\right],\left|f_{t}\right| \leq \epsilon}\left|f_{0}\right|^{\alpha \delta}\left|f_{t}\right|^{\alpha(1-\delta)} d \mu \\
& =I_{1}+I_{2}+I_{3} \text {. }
\end{aligned}
$$

By Hölder's inequality,

$$
I_{2} \leq\left(\int_{\left|f_{0}\right| \notin\left[\eta, \eta^{-1}\right]}\left|f_{0}\right|^{\alpha} d \mu\right)^{\delta}\left(\int_{S}\left|f_{t}\right|^{\alpha} d \mu\right)^{1-\delta}
$$

hence, by choosing $\eta$ sufficiently small, we make $I_{2}$ small uniformly in $t$. Since

$$
I_{3} \leq\left(\epsilon \eta^{-1}\right)^{\alpha(1-\delta)} \int_{S}\left|f_{0}\right|^{\alpha} d \mu
$$


we can choose $\epsilon$ small to make $I_{3}$ small uniformly in $t$. Having $\epsilon$ and $\eta$ fixed, we obtain by Hölder's inequality and (2.5)

$$
\limsup _{t \rightarrow \infty} I_{1} \leq \lim _{t \rightarrow \infty}\left(\int_{\left|f_{0}\right| \in\left[\eta, \eta^{-1}\right],\left|f_{t}\right|>\epsilon}\left|f_{0}\right|^{\alpha} d \mu\right)^{\delta}\left(\int_{S}\left|f_{t}\right|^{\alpha} d \mu\right)^{1-\delta}=0 .
$$

This completes the proof of (2.4). To prove the converse we notice that Markov's inequality and (2.4) imply (2.5) for $t \rightarrow \infty$. Since

$$
\int_{S}\left|f_{0}\right|^{\alpha(1-\delta)}\left|f_{-t}\right|^{\alpha \delta} d \mu=\int_{S}\left|f_{0}\right|^{\alpha \delta}\left|f_{t}\right|^{\alpha(1-\delta)} d \mu
$$

the same argument gives (2.5) when $t \rightarrow-\infty$. The proof is complete.

Remark 2.1 Alternatively, one can derive Proposition 2.1 directly from Theorem 2 of Cambanis, Hardin and Weron, [CHW87]. However, that way seems to be more tedious than a simple use of (2.5) above.

The following theorem characterizes the flows on finite measure spaces generating mixing $\mathrm{S} \alpha \mathrm{S}$ processes.

Theorem 2.1 Let $\boldsymbol{X}=\left\{X_{t}\right\}_{t \in \mathbf{T}}$ be a stationary $S \alpha S$ process with representation (1.1)-(1.3). Assume $\mu(S)<\infty$. Then the following conditions are equivalent

(a) $\boldsymbol{X}$ is mixing;

(b) $\left\{\phi_{t}\right\}_{t \in \mathbf{T}}$ is asymptotically singular.

Proof: Since $\mu \circ \phi_{t}$ and $\mu$ are mutually absolutely continuous, we can take $\lambda=\mu$ in (2.2), thus

$$
H\left(\mu, \mu \circ \phi_{t}\right)=\int_{S}\left(\frac{d\left(\mu \circ \phi_{t}\right)}{d \mu}\right)^{1 / 2} d \mu .
$$

We will use Proposition 2.1 with $\delta=1 / 2$. Since for every $r, t$

$$
\int_{S}\left|f_{r} f_{r+t}\right|^{\alpha / 2} d \mu=\int_{S}\left|f_{0} f_{t}\right|^{\alpha / 2} d \mu
$$

we get from (2.4)

$$
\lim _{t \rightarrow \infty} \int_{S}\left|f_{r} f_{t}\right|^{\alpha / 2} d \mu=0 \quad \text { for each } r
$$

By (1.3) and the finiteness of $\mu$, for every $\epsilon>0$ there exists a finite set $t_{1}, \ldots, t_{n} \in \mathbf{T}$ and $\delta>0$ such that

$$
\mu\left(S \backslash \bigcup\left\{\left|f_{t_{i}}\right|>\delta\right\}\right)<\epsilon .
$$


Consider

$$
g=\sum\left|f_{t_{i}}\right|
$$

and

$$
g_{t}=\sum\left|f_{t_{i}+t}\right|=\left(\frac{d\left(\mu \circ \phi_{t}\right)}{d \mu}\right)^{1 / \alpha} g \circ \phi_{t}
$$

From (2.6) we get

$$
\limsup _{t \rightarrow \infty} \int_{S}\left|g g_{t}\right|^{\alpha / 2} d \mu \leq \sum_{i, j} \lim _{t \rightarrow \infty} \int_{S}\left|f_{t_{i}} f_{t_{j}+t}\right|^{\alpha / 2} d \mu=0 .
$$

Let $A=\{|g|>\delta\} ;$ by $(2.7), \mu(S-A)<\epsilon$. We obtain

$$
\begin{gathered}
H\left(\mu, \mu \circ \phi_{t}\right) \leq \int_{A \cap \phi_{t}^{-1} A}\left(\frac{d\left(\mu \circ \phi_{t}\right)}{d \mu}\right)^{1 / 2} d \mu \\
+\int_{A^{c}}\left(\frac{d\left(\mu \circ \phi_{t}\right)}{d \mu}\right)^{1 / 2} d \mu+\int_{\phi_{t}^{-1} A^{c}}\left(\frac{d\left(\mu \circ \phi_{t}\right)}{d \mu}\right)^{1 / 2} d \mu \\
\leq \delta^{-\alpha} \int_{S}\left|g g_{t}\right|^{\alpha / 2} d \mu+2 \mu\left(A^{c}\right)^{1 / 2} \mu(S)^{1 / 2} .
\end{gathered}
$$

Thus

$$
\limsup _{t \rightarrow \infty} H\left(\mu, \mu \circ \phi_{t}\right) \leq 2 \mu(S)^{1 / 2} \epsilon^{1 / 2},
$$

proving (b) after letting $\epsilon$ go to zero.

The converse is easy. For any $M>0$ we have

$$
\begin{gathered}
\int_{S}\left|f_{0} f_{t}\right|^{\alpha / 2} d \mu \leq \int_{\left|f_{0}\right|>M}\left|f_{0} f_{t}\right|^{\alpha / 2} d \mu \\
+\int_{\left|f_{0} \circ \phi_{t}\right|>M}\left|f_{0} f_{t}\right|^{\alpha / 2} d \mu+M^{\alpha} \int_{S}\left(\frac{d\left(\mu \circ \phi_{t}\right)}{d \mu}\right)^{1 / 2} d \mu \\
\leq 2\left(\int_{\left|f_{0}\right|>M}\left|f_{0}\right|^{\alpha} d \mu\right)^{1 / 2}\left(\int_{S}\left|f_{0}\right|^{\alpha} d \mu\right)^{1 / 2}+M^{\alpha} \int_{S}\left(\frac{d\left(\mu \circ \phi_{t}\right)}{d \mu}\right)^{1 / 2} d \mu .
\end{gathered}
$$

Taking $M$ sufficiently large and then letting $t \rightarrow \infty$ we prove (2.4), and so (a).

Remark 2.2 The assumption (1.3) is not needed for the implication (b) $\Rightarrow$ (a): asymptotic singularity gives mixing. 
Despite the fact that one can always choose a representation (1.1) with finite measure $\mu$, in certain cases the natural representation for a $\mathrm{S} \alpha \mathrm{S}$ process involves an infinite measure, and so it is useful to have conditions for mixing when $\mu$ is arbitrary.

Theorem 2.2 Let $\boldsymbol{X}=\left\{X_{t}\right\}_{t \in \mathbf{T}}$ be a stationary $S \alpha S$ process with representation (1.1)-(1.3). Then the following conditions are equivalent

(a) $\boldsymbol{X}$ is mixing;

(b) for every $A \subset S$ of finite measure $\mu$,

$$
\lim _{t \rightarrow \infty} \int_{A \cap \phi_{t} A}\left(\frac{d\left(\mu \circ \phi_{t}\right)}{d \mu}\right)^{1 / 2} d \mu=0
$$

(c) there exist $A_{k} \nearrow S$ such that for each $k \geq 1$,

$$
\lim _{t \rightarrow \infty} \int_{A_{k} \cap \phi_{t} A_{k}}\left(\frac{d\left(\mu \circ \phi_{t}\right)}{d \mu}\right)^{1 / 2} d \mu=0
$$

Moreover, the implication (c) $\Rightarrow($ a) holds without assumption (1.3).

Proof: Choose $h>0$ such that $\int_{S} h^{\alpha} d \mu=1$ and define

$$
f_{t}^{*}=\frac{f_{t}}{h}
$$

$\left\{f_{t}^{*}\right\}$ is another representation of $\boldsymbol{X}$ with respect to a random measure $M^{*}$ with control measure $\mu^{*}$ such that $d \mu^{*}=h^{\alpha} d \mu$. Since $f_{t}$ is given by (1.2),

$$
f_{t}^{*}=a_{t}\left(\frac{d\left(\mu^{*} \circ \phi_{t}\right)}{d \mu^{*}}\right)^{1 / \alpha} f^{*} \circ \phi_{t} .
$$

Thus, by Theorem $2.1, \boldsymbol{X}$ is mixing if and only if $\left\{\phi_{t}\right\}$ is asymptotically singular with respect to the probability measure $\mu^{*}$. Assume (a) and let $A$ be such that $\mu(A)<\infty$. We have

$$
\begin{gathered}
\int_{A \cap \phi_{t} A}\left(\frac{d\left(\mu \circ \phi_{t}\right)}{d \mu}\right)^{1 / 2} d \mu \\
=\int_{S}\left(\mathbf{1}_{A} h^{-\alpha / 2}\right)\left[\left(\mathbf{1}_{A} h^{-\alpha / 2}\right) \circ \phi_{t}\right]\left(\frac{d\left(\mu^{*} \circ \phi_{t}\right)}{d \mu^{*}}\right)^{1 / 2} d \mu^{*} \\
\leq \int_{h^{-\alpha / 2}>M}\left(\mathbf{1}_{A} h^{-\alpha / 2}\right)\left[\left(\mathbf{1}_{A} h^{-\alpha / 2}\right) \circ \phi_{t}\right]\left(\frac{d\left(\mu^{*} \circ \phi_{t}\right)}{d \mu^{*}}\right)^{1 / 2} d \mu^{*}
\end{gathered}
$$




$$
\begin{gathered}
+\int_{h^{-\alpha / 2} \circ \phi_{t}>M}\left(\mathbf{1}_{A} h^{-\alpha / 2}\right)\left[\left(\mathbf{1}_{A} h^{-\alpha / 2}\right) \circ \phi_{t}\right]\left(\frac{d\left(\mu^{*} \circ \phi_{t}\right)}{d \mu^{*}}\right)^{1 / 2} d \mu^{*} \\
+M^{2} \int_{S}\left(\frac{d\left(\mu^{*} \circ \phi_{t}\right)}{d \mu^{*}}\right)^{1 / 2} d \mu^{*} \\
\leq 2 \mu\left(A \cap\left\{h^{-\alpha / 2}>M\right\}\right)^{1 / 2} \mu(A)^{1 / 2}+M^{2} \int_{S}\left(\frac{d\left(\mu^{*} \circ \phi_{t}\right)}{d \mu^{*}}\right)^{1 / 2} d \mu^{*} .
\end{gathered}
$$

Letting $t \rightarrow \infty$ and then $M \rightarrow \infty$ we complete the proof of (b).

Obviously (b) $\Rightarrow$ (c). Assume (c) (and (1.1)-(1.2), but not (1.3)). Using the fact that $\mu^{*}$ is a probability measure, we obtain for each $k \geq 1$,

$$
\begin{gathered}
\int_{S}\left(\frac{d\left(\mu^{*} \circ \phi_{t}\right)}{d \mu^{*}}\right)^{1 / 2} d \mu^{*} \\
\leq \int_{A_{k} \cap \phi_{t} A_{k}}\left(\frac{d\left(\mu^{*} \circ \phi_{t}\right)}{d \mu^{*}}\right)^{1 / 2} d \mu^{*}+\int_{A_{k}^{c}}\left(\frac{d\left(\mu^{*} \circ \phi_{t}\right)}{d \mu^{*}}\right)^{1 / 2} d \mu^{*} \\
+\int_{\phi_{t} A_{k}^{c}}\left(\frac{d\left(\mu^{*} \circ \phi_{t}\right)}{d \mu^{*}}\right)^{1 / 2} d \mu^{*} \\
\leq \int_{A_{k} \cap \phi_{t} A_{k}}\left(\frac{d\left(\mu^{*} \circ \phi_{t}\right)}{d \mu^{*}}\right)^{1 / 2} d \mu^{*}+2 \mu^{*}\left(A_{k}^{c}\right)^{1 / 2} .
\end{gathered}
$$

Letting $t \rightarrow \infty$ and then $k \rightarrow \infty$ gives (b) of Theorem 2.1. This implies (a) by Remark 2.2 and ends the proof.

Probably the most interesting case of Theorem 2.2 is when $\mu$ is an infinite measure invariant under $\left\{\phi_{t}\right\}$. Since the Radon-Nikodym derivative equals 1 , we obtain the following corollary, equivalent to Theorem 4.1 of [Gro94].

Corollary 2.1 Under the assumptions of Theorem 2.2 suppose that $\mu$ is invariant under $\left\{\phi_{t}\right\}_{t \in \mathbf{T}}$. Then the following conditions are equivalent

(a) $\boldsymbol{X}$ is mixing;

(b) for every $A \subset S$ of finite measure $\mu$,

$$
\lim _{t \rightarrow \infty} \mu\left(A \cap \phi_{t} A\right)=0
$$

(c) there exist $A_{k} \nearrow S$ such that for each $k \geq 1$,

$$
\lim _{t \rightarrow \infty} \mu\left(A_{k} \cap \phi_{t} A_{k}\right)=0 .
$$


Moreover, the implication (c) $\Rightarrow$ (a) holds without assumption (1.3).

Remark 2.3 As observed by A. Gross [Gro94], restricting $t$ in (2.5) to a set of density one (which may depend on $K$ and $\epsilon$ ) gives the weak mixing of the process instead of mixing. Since our computations do not depend on whether $t$ runs over the entire set $\mathbf{T}$ or only over its infinite subset, we conclude that adding the phrase

"there exists a set $D \subset \mathbf{T}$ of density one"

in the statements characterizing mixing in this section we obtain corresponding results on ergodicity (and weak mixing) of $\mathrm{S} \alpha \mathrm{S}$ processes.

The last part of this section (as well as the next one) is devoted to S $\alpha \mathrm{S}$ processes of the type $\boldsymbol{X}^{3}$ (recall the decomposition (1.4)). As we have mentioned in the Introduction, for such processes to be mixing (or even ergodic) it is necessary to be such in spite of the fact that the flow $\left\{\phi_{t}\right\}$ is infinitely recurrent, and so generates "memory" within the process. It turns out that the flow should not return "too often" to the starting point, and we start now making this statement precise. The most logical way of doing so is, of course, through the expected return time.

Suppose that $\mu$ is invariant under $\left\{\phi_{t}\right\}$. The return time to a set $A \in \mathcal{B}$ is defined by $\tau_{A}(s)=\inf \left\{t>0: \phi_{t}(s) \in A\right\} \quad(s \in A)$ and, if $\mu(A)<\infty$,

$$
\frac{1}{\mu(A)} \int_{A} \tau_{A} d \mu
$$

is the expected return time. To avoid measurability problems we consider the case $\mathbf{T}=\mathbf{Z}$. Under these assumptions we obtain the following

Proposition 2.2 Suppose that $\boldsymbol{X}^{3}$ is ergodic. Then the expected return time is infinite for any set $A$ of a finite positive measure.

Proof. Kac's theorem (see [Kre85], p. 19) gives

$$
\int_{A} \tau_{A} d \mu=\mu\left(\cup_{n=0}^{\infty} \phi_{-n}(A)\right)
$$

Let $\delta=\mu(A)>0$. Since by Corollary 2.1

$$
\mu\left(A \cap \phi_{-n}(A)\right)=\mu\left(A \cap \phi_{n}(A)\right) \rightarrow 0,
$$

as $n \rightarrow \infty$, for every $\theta>0$ there is an $N$ such that for every $n>N$

$$
\mu\left(A \cap \phi_{-n}(A)\right)<\theta .
$$


For every $k \geq 1$ we have

$$
\begin{gathered}
\mu\left(\cup_{n=0}^{\infty} \phi_{-n}(A)\right) \geq \mu\left(\cup_{n=0}^{k} \phi_{-n N}(A)\right) \\
\geq \sum_{n=0}^{k} \mu\left(\phi_{-n N}(A)\right)-\sum_{n=0}^{k} \sum_{m=n+1}^{k} \mu\left(\phi_{-n N}(A) \cap \phi_{-m N}(A)\right) \\
=(k+1) \mu(A)-\sum_{n=0}^{k} \sum_{m=n+1}^{k} \mu\left(A \cap \phi_{-(m-n) N}(A)\right) \\
\geq(k+1) \delta-2^{-1} k(k+1) \theta .
\end{gathered}
$$

Letting first $\theta \rightarrow 0$ and then $k \rightarrow \infty$, we see immediately that the right hand side of (2.8) is infinite.

\section{Mixing $\mathbf{S} \alpha \mathbf{S}$ processes generated by conserva- tive flows}

In this section we will show that mixing stationary $S \alpha S$ processes of the type $\boldsymbol{X}^{3}$ really exist. For simplicity we assume $\mathbf{T}=\mathbf{Z}$, but see the construction at the end of the section.

Consider a bilateral real valued Markov chain $\left\{S_{n}\right\}_{n \in \mathbf{Z}}$ defined on the canonical coordinate space $S=\mathbf{R}^{\mathbf{Z}}$ with a stationary transition probability function

$$
Q(x, B)=\operatorname{Pr}\left(S_{n+1} \in B \mid S_{n}=x\right) \text {. }
$$

Suppose that $m$ is an invariant measure for $\left\{S_{n}\right\}_{n \in \mathbf{Z}}$. That is, $m$ is a Radon measure on $\mathbf{R}$ such that

$$
m(B)=\int_{\mathbf{R}} Q(x, B) m(d x), \quad \forall B \in \mathcal{B}_{\mathbf{R}} .
$$

Denoting by $Q^{x}$ be the distribution of $\left\{S_{n}\right\}_{n \in \mathbf{Z}}$ as a random vector in $\left(S, \mathcal{B}_{S}\right)$ starting from $x \in \mathbf{R}$ (i.e., $Q^{x}\left\{S_{0}=x\right\}=1$ ), we define a measure $\mu$ on $\left(S, \mathcal{B}_{S}\right)$ by

$$
\mu(A)=\int_{\mathbf{R}} Q^{x}(A) m(d x), \quad A \in \mathcal{B}_{S} .
$$

Then, under (possibly infinite) law $\mu,\left\{S_{n}\right\}$ can be viewed as a stationary Markov chain with the initial "distribution" $m$. We suppose that $\left\{S_{n}\right\}$ is $m$-recurrent. That is, $\forall B \in \mathcal{B}_{\mathbf{R}}$ with $m(B)>0$,

$$
\operatorname{Pr}\left(S_{n} \in B \text { infinitely often } \mid S_{0}=x\right)=1 \quad \text { for } m-\text { a.a. } x \in \mathbf{R} \text {. }
$$

See, e.g., Orey [Ore71], p.4. We have the following 
Proposition 3.1 The shift transformation $\theta$ on $S$ is measure preserving, conservative, and the following are equivalent

(A) the measure $\mu$ is infinite;

(B) the invariant measure $m$ is infinite;

(C) $\forall$ bounded set $B \in \mathcal{B}_{\mathbf{R}}$

$$
\lim _{n \rightarrow \infty} \operatorname{Pr}\left(S_{n} \in B \mid S_{0}=x\right)=0 \quad \text { for } m-\text { a.a. } x \in \mathbf{R} .
$$

Furthermore, under any of the above, the flow $\phi_{n}:=\theta^{n}, n \in \mathbf{Z}$ satisfies (c) of Corollary 2.1.

Proof: The fact that $\theta$ preserves measure $\mu$ follows simply from invariance of the measure $m$, while the fact that it is conservative follows from Harris and Robbins [HR53].

The equivalence of $(A)$ and $(B)$ is trivial, as well as the fact that $(C)$ implies $(B)$. The implication $(B) \Rightarrow(C)$ follows from Theorem 7.3 of Orey [Ore71].

It remains to show that (3.3) implies $(c)$ of Corollary 2.1. Recall that $S_{n}$ is the projection onto the $n$th coordinate of $S$ and let

$$
A_{k}=\left\{\left|S_{0}\right| \leq k\right\}
$$

Then

$$
\begin{gathered}
\mu\left(A_{k} \cap \phi_{n} A_{k}\right)=\mu\left(\theta^{-n} A_{k} \cap A_{k}\right) \\
=\int_{[-k, k]} \operatorname{Pr}\left(S_{n} \in[-k, k] \mid S_{0}=x\right) m(d x) \rightarrow 0
\end{gathered}
$$

as $n \rightarrow \infty$ by (3.3), completing the proof.

As an immediate consequence of Proposition 3.1 we obtain the following class of mixing stationary $\mathrm{S} \alpha \mathrm{S}$ processes generated by conservative flows.

Corollary 3.1 Under the above assumptions suppose that the invariant measure $m$ is infinite. Let $f \in L^{\alpha}(S, \mu)$ and let $a_{n}: S \rightarrow\{-1,1\}$ satisfies $a_{n+m}=a_{n} \cdot a_{m} \circ \theta^{n}$ $\mu-a . e$. Then the stationary $S \alpha S$ process

$$
X_{n}=\int_{S} a_{n} f \circ \theta^{n} d M
$$

is of type $\boldsymbol{X}^{3}$, and is mixing. 


\section{Remarks}

(i) The simplest choice of parameters in Corollary 3.1 that produces a rich class of processes is to take $a_{n} \equiv 1$, and $f\left(\ldots, x_{-1}, x_{0}, x_{1}, \ldots\right)=\mathbf{1}\left(x_{0} \in A\right)$ for a Borel subset $A$ of $\mathbf{R}$.

(ii) Processes of this form belong to the class of doubly stationary processes (see [CHW87] and [GW94]).

(iii) The connection described above between the recurrence properties of a Markov chain $\left\{S_{n}\right\}$ and ergodic properties of the stationary $\mathrm{S} \alpha \mathrm{S}$ process $\left\{X_{n}\right\}$ becomes especially transparent if the Markov chain is, say, integer valued. Then, if it is null recurrent, then (3.3) holds by Theorem 69 (b) in Freedman [Fre83], p.25, and so the stationary $\mathrm{S} \alpha \mathrm{S}$ process is mixing. On the other hand, if the Markov chain is positive recurrent, then the invariant measure $m$ is finite, and then so is the measure $\mu$, implying that the $\mathrm{S} \alpha \mathrm{S}$ process is not even ergodic, as was observed above. This is another demonstration of the phenomenon presented in Proposition 2.2: mixing (or even ergodicity) of a $\mathrm{S} \alpha \mathrm{S}$ process requires the underlying flows to have infinite expected return times.

(iv) The simplest case when the conditions for mixing of (3.4) are easy to check is the case of a random walk $\left\{S_{n}\right\}$. Let $F$ be the common distribution of $S_{n+1}-S_{n}$. It is then well known that $\left\{S_{n}\right\}$ is $m$-recurrent (with $m$ being the Lebesgue measure if $F$ is not concentrated on any lattice $L_{d}=\{n d: n \in \mathbf{Z}\}$, and $m$ being the counting measure on $L_{d}$ if $F$ is concentrated on it) if and only if the characteristic function $\hat{F}$ of $F$ satisfies

$$
\lim _{s \rightarrow 1^{+}} \int_{-1}^{1} \Re \frac{1}{1-s \hat{F}(u)} d u=\infty
$$

(see Chung and Fuchs [CF51], or Feller [Fel71] (XVII.6)). If the random walk is actually concentrated on $L_{d}$, then an equivalent condition is

$$
\int_{-1}^{1} \Re \frac{1}{1-\hat{F}(u)} d u=\infty
$$

(Spitzer [Spi64]). If $\int_{\mathbf{R}}|x| F(d x)<\infty$, then an easier to verify necessary and sufficient condition for recurrence is $\int_{\mathbf{R}} x F(d x)=0$, (see [Fel71], VI.10).

Of course, for a recurrent random walk the condition (3.3) follows by the concentration inequality for sums of independent random variables. Therefore, Markov shifts corresponding to recurrent random walks generate mixing $\mathrm{S} \alpha \mathrm{S}$ processes of the type $\boldsymbol{X}^{3}$.

The last remark above has an obvious extension to the continuous time $\mathbf{T}=\mathbf{R}$. We replace a random walk by a bilateral Lévy process $\left\{S_{t}\right\}_{t \in \mathbf{R}}$ with stationary independent increments (e.g. Wiener process) having sample paths in $S:=D(\mathbf{R})$. In this 
case the invariant initial measure $m$ is either the counting measure on a lattice $L_{d}$ or the Lebesgue measure on $\mathbf{R}$, depending on whether $F_{t}:=\mathcal{L}\left\{S_{u+t}-S_{u}\right\}, t, u \in \mathbf{R}$ are all concentrated on the lattice $L_{d}$, or, whether the latter condition does not hold. The usual notion of recurrence in this context means that $P\left\{\overline{\lim }_{t \rightarrow \infty} \mathbf{1}_{G}\left(S_{t}\right)=1\right\}=1$ for every open neighborhood $G$ of the origin and transience means that $P\left\{\lim _{t \rightarrow \infty}\left|S_{t}\right|=\right.$ $\infty\}=1$. It follows from Kingman [Kin64] that $\left\{S_{t}\right\}_{t \geq 0}$ is recurrent (transient) if and only if the discrete random walk $\left\{S_{n}\right\}_{n \in \mathbf{Z}^{+}}$is recurrent (transient).

Defining a measure $\mu$ on $S=D(\mathbf{R})$ by (3.1), where as before, $Q^{x}$ is the distribution on $S$ of $\left\{S_{t}\right\}_{t \in \mathbf{R}}$ starting from $x$, one observes, as above, that the $\mu$ satisfies (c) of Corollary 2.1 with $\phi_{t}$ defined as the shift on $S,\left(\phi_{t}(s)\right)_{u}=s_{u+t}$. Therefore, any S $\alpha S$ process given by

$$
X_{t}=\int_{S} a_{t} f \circ \phi_{t} d M, t \in \mathbf{R},
$$

is mixing and of type $\boldsymbol{X}^{3}$. Here $f \in L^{\alpha}(S, \mu)$ and $a: S \rightarrow\{-1,1\}$ satisfies $a_{t_{1}+t_{2}}=$ $a_{t_{2}} a_{t_{1}} \circ \phi_{t_{2}} \mu$ a.e., for every $t_{1}, t_{2} \in \mathbf{R}$.

\section{References}

[CF51] K.L. Chung and W.H.J. Fuchs. On the distribution of values of sums of random variables. Mem. Amer. Math. Soc., 6, 1951.

[CHW87] S. Cambanis, C.D. Hardin, Jr., and A. Weron. Ergodic properties of stationary stable processes. Stochastic Proc. Appl., 24:1-18, 1987.

[Fel71] W. Feller. An Introduction to Probability Theory and its Applications, volume 2. Wiley, New York, 2nd edition, 1971.

[Fre83] D. Freedman. Markov Chain. Springer-Verlag, New York, 1983.

[GR93] A. Gross and J.B. Robertson. Ergodic properties of random measures on stationary sequences of sets. Stoch. Proc. Appl., 46:249--265, 1993.

[Gro94] A. Gross. Some mixing conditions for stationary symmetric stable stochastic processes. Stoch. Proc. Appl., 51:277-295, 1994.

[GW94] A. Gross and A. Weron. On measure-preserving transformations and spectral representations of stationary symetric stable processes. Technical report, 1994.

[Har82] C.D. Hardin Jr. On the spectral representation of symmetric stable processes. J. Mult. Anal, 12:385-401, 1982. 
[HR53] T.E. Harris and H. Robbins. Ergodic theory of markov chains admitting an infinite invariant measure. Proc. Natl. Acad. Sci., 39:860-864, 1953.

[Kin64] J.F.C. Kingman. Recurrence properties of processes with stationary independent increments. J. Austral. Math. Soc., 4:223-228, 1964.

[Kre85] U. Krengel. Ergodic Theorems. De Gruyter, Berlin, New York, 1985.

[Mar70] G. Maruyama. Infinitely divisible processes. Theory Prob. Appl., 15(1):122,1970 .

[Ore71] S. Orey. Limit Theorems for Markov Chain Transition Probabilties. Van Nostrand Reinold, London, 1971.

[Pet83] K. Petersen. Ergodic Theory. Cambridge University Press, Cambrdge, 1983.

[Pod92] K. Podgórski. A note on ergodic symmetric symmetric stable processes. Stochastic Processes and their Applications, 43:355-362, 1992.

[Ros95] J. Rosiński. On the structure of stationary stable processes. Ann. Probab., 23:--, 1995.

[Spi64] F. Spitzer. Principle of Random walk. D. van Nostrand Company, Princeton, 1964.

[SRMC93] D. Surgailis, J. Rosiński, V. Mandrekar, and S. Cambanis. Stable mixed moving averages. Probab. Theory Related Fields, 97:543-558, 1993.

[ST94] G. Samorodnitsky and M.S. Taqqu. Stable Non-Gaussian Random Processes. Chapman and Hall, New York, 1994. 